\title{
Stem Cells-Cancer Research
}

\section{Vijaya Krishna Varanasi*}

Post doctoral research, Washington State University, USA

\section{Editorial}

Open access refers to unrestricted access via the internet to articles published in scholarly journals such as "Journal of Carcinogenesis \& Mutagenesis". The main benefit of open access journals is that they are integrated with search engines, such as Google Scholar and anyone or anywhere connected to the web will have free access to their topic of interest. Open Access articles are cited much more than the non open access articles [1] and have greater visibility in the scientific community and public. In medical and pharmaceutical sciences it will enable doctors, pharmacists and clinical researchers to increase their medical knowhow and share it with their colleagues for the advancement of their field of study. OMICS Publishing Group strongly supports this open access initiative and all articles published by OMICS Publishing Group are freely accessible to everyone immediately after publication. Some of the special features of OMICS group journals include digital formatting, audio listening, language translation and ability to share views on articles via social networking. According to a recent report $88 \%$ of respondents believed that publicly funded research should be made available to be read and used without barriers to access [2]. The report also revealed that about $94 \%$ of cancer researchers use internet for their professional activities every day and sharing of information can accelerate progress in the scientific battle against cancer. The "Journal of Carcinogenesis and Mutagenesis" focuses on the recognition of cellular responses to DNA damage, apoptosis (cell death), including the inactivation of tumor suppressor genes, and analysis of carcinogenic process by genetic and epigenetic alterations in genes for the study of cancer initiation and progression.

Advances in cancer biology in the last couple of years have provided us with new insights into the origin of tumors and metastatic potential of cancer. The discovery of cancer stem cells (CSCs) has increased our understanding of the complexities of cancer therapy. Studies conducted in the last decade or so have confirmed the presence of subpopulations of cells known as cancer stem cells within tumors that drive tumor growth and recurrence [3]. CSCs are known to possess three major characteristics: (1) they can trigger initiation of tumors and drive neoplastic proliferation, (2) capacity to self-renewal, and (3) CSCs can differentiate into more mature non-stem cell like cancer cells $[4,5]$. According to the current version of cancer stem cell hypothesis, only a portion of the tumor cells are responsible for driving and maintaining tumorous growth in the tissues. It is this subset of cells that need to be targeted and destroyed by agents in order to achieve any successful cure for cancers.

The idea that cancer arises from stem cells was first proposed over 150 years ago as the embryonal rest theory of cancer [6]. By the beginning of the 20th century, the embryonal rest theory of cancer was rejected and was replaced by the concept of de-differentiation of cancer cells. Two major recent observations led to the theory that cancers could be maintained by stem cells [7]. Tumors are heterogeneous in their ability to give rise to more tumors and that tumors exhibit similarities to the derived tissue in terms of histological and molecular stand points. This suggested a hierarchical relationship among tumor cells similar to what we observe in normal healthy tissues. A large number of existing therapies for cancer induce cell death by causing DNA damage without affecting the normal stem cells. Normal stem cells are resistant to various cytotoxic agents causing cell death. The obvious similarities between normal stem cells and CSCs led to the hypothesis that CSCs may also be resistant to common chemical agents [4] and that improvement in treating cancers can be attained by directly targeting CSCs. Numerous studies have reported that "cancer stem cell-like cells" present in some established cell lines are resistant to cytotoxic agents $[8,9]$. Therefore, the most effective therapeutic strategy would be to directly target the survival, self-renewal capacity and other key pathways of CSCs while causing minimum damage to normal cells. In order to achieve these objectives efforts should be made to identify the critical survival and self-renewal pathways that are active in CSCs and find ones that do not overlap with critical normal tissues and especially their stem cells.

One of the approaches to eradicate CSCs that is gaining increasing interest is the use of oncolytic adenoviruses. Oncolytic adenoviruses Ad5/3-Delta24 and Ad5.pk7-Delta24 can be effective against CSCs such as CD44+CD24-/low cells that were identified in breast cancer patients [10]. It was also reported that brain CSCs were susceptible to adenovirus-mediated cell death by autophagy [11]. Study of Wnt signaling pathway and its various components is gaining focus in the research community [12]. Wnt pathway was found to be involved in the fate of stem cells [13] and its role as a regulator of stem cell choice to proliferate or self-renew. Given these properties, it is not surprising that a strong correlation between Wnt signaling and the onset of cancer exist. Wnt signaling was found to be active during tumor development and knockdown of Wnt 2 and the mediator $\beta$-catenin by siRNA in human U251 glioma cells suppressed cell proliferation and caused cell death. Currently, there are many investigations underway to target various components of the Wnt pathway as a means of finding more effective treatments for cancer.

A well known CSC marker, CD133 is a key therapeutic target for metastatic melanoma and for other CD133-expressing cancer types [14]. It was reported that cells fail to metastasize once CD133 expression goes down. In all tissues, stem cells are located in specific niches [15] and targeting these niches is another promising therapeutic option.

*Corresponding author: Vijaya Krishna Varanasi, Post doctoral research, Washington State University, USA, E-mail: vijaya.varanasi@gmail.com

Received December 22, 2011; Accepted December 26, 2011; Published December 28, 2011

Citation: Vijaya Krishna V (2011) Stem Cells-Cancer Research. J Carcinogene Mutagene S1:e001. doi:10.4172/2157-2518.S1-e001

Copyright: ( 2011 Vijaya Krishna V. This is an open-access article distributed under the terms of the Creative Commons Attribution License, which permits unrestricted use, distribution, and reproduction in any medium, provided the original author and source are credited. 
MicroRNAs (miRNA) are non-coding RNAs that have emerged as key post-transcriptional regulators of gene expression in various species with promising results in cancer therapy [16].

Research efforts should be made to better understand CSCs in 3D cell culture. Tissues and organs are three dimensional (3D) structures. But we have understood various aspects of organ formation, their function, and pathology based on available two-dimensional (2D) cell cultures or on animal model systems [17]. The 2D cell cultures can differ significantly in their morphology, cell-to-cell, and cellmatrix interactions from those growing in 3D environments [18]. Animal models on the other hand may fail to replicate human tumors, drug responses, autoimmune diseases, and stem cell differentiation. Therefore, 3D models fill the gap perfectly between existing 2D models and animal models [19]. The models such as 3D spheroids also provide an alternative approach to better understand the resistance mechanisms in tumor biology and for evaluating efficacy of tumor therapeutic agents [20].

Over the past few decades, tremendous amount of knowledge in the area of CSC biology has been gained by scientific community. But not until recently scientists were able to unravel the mysteries of CSCs. Lot of questions are still unanswered. It is a puzzle why some tumor cells are more or less tumorigenic than others. Cancer researchers worldwide should also need to confront issues such as limited number of CSCs in tumor tissues, technical difficulties in keeping CSCs in any culture, and their aberrant strong drug resistance. A better understanding of the differences between normal and cancer stem cells may help in revealing new targets for developing innovative treatments for cancer. Integration of various cytotoxic, biologic, and CSC-directed therapies will allow rapid improvements in patient outcomes. I am sure the "Journal of Carcinogenesis \& Mutagenesis" from OMICS Group will play an important role in addressing some of these key issues in cancer stem cell research.

\section{References}

1. Eysenbach G (2006) The open access advantage. J Med Internet Res 8: 8.

2. Kenney R, Warden R (2011) An Open Access future? Report from the eurocancercoms project. ecancer 5: 223.

3. Al-Hajj M, Wicha MS, Benito-Hernandez A, Morrison SJ, Clarke MF (2003) Prospective identification of tumorigenic breast cancer cells. Proc Natl Acad Sci USA 100: 3983-3988.

4. Reya T, Morrison SJ, Clarke MF, Weissman IL (2001) Stem cells, cancer, and cancer stem cells. Nature 414: 105-111.

5. Schatton T, Frank NY, Frank MH (2009) Identification and targeting of cancer stem cells. Bioessays 10: 1038-1049.

6. Potter VR (1978) Phenotypic diversity in experimental hepatomas: the concept of partially blocked ontogeny. The 10th Walter Hubert Lecture. $\mathrm{Br} \mathrm{J}$ Cancer 38: 1-23.

7. Diehn M, Cho RW, Clarke MF (2009) Therapeutic Implications of the Cancer Stem Cell Hypothesis. Semin Radiat Oncol 19: 78-86.

8. Todaro M, Alea MP, Di Stefano AB, Cammareri P, Vermeulen L, et al. (2007) Colon cancer stem cells dictate tumor growth and resist cell death by production of interleukin-4. Cell Stem Cell 1: 389-402.
9. Ho MM, Ng AV, Lam S, Hung JY (2007) Side population in human lung cancer cell lines and tumors is enriched with stem-like cancer cells. Cancer Res 67: 4827-4833.

10. Eriksson M, Guse K, Bauerschmitz G, Virkkunen P, Tarkkanen M, et al. (2007) Oncolytic adenoviruses kill breast cancer initiating CD44+CD24-/low cells. Mol Ther 15: 2088-2093.

11. Jiang H, Gomez-Manzano C, Aoki H, Alonso MM, Kondo S, et al. (2007) Examination of the therapeutic potential of Delta-24-RGD in brain tumor stem cells: role of autophagic cell death. J Natl Cancer Inst 99: 1410-1414.

12. Woodward WA, Chen MS, Behbod F, Alfaro MP, Buchholz TA (2007) WNT/B catenin mediates radiation resistance of mouse mammary progenitor cells. Proc Natl Acad Sci USA 104: 618-623.

13. Roel N (2005) Wnt Signaling in Disease and Development. Cell Res 15: 28-32.

14. Rappa G, Fodstad O, Lorico A (2008) The stem cell-associated antigen CD133 (Prominin-1) is a molecular therapeutic target for metastatic melanoma. Stem Cells 26: 3008-3017.

15. Alison MR, Islam S (2009) Attributes of adult stem cells. J Pathol 217: 144-160.

16. Hiratsuka S, Watanabe A, Aburatani H, Maru Y (2006) Tumourmediated upregulation of chemoattractants and recruitment of myeloid cells predetermines lung metastasis. Nat Cell Biol 8: 1369-1375.

17. Yamada KM, Cukierman E (2007) Modeling tissue morphogenesis and cancer in 3D. Cell 130: 601-610.

18. Griffith LG, Swartz MA (2006) Capturing complex 3D tissue physiology in vitro. Nat Rev Mol Cell Biol 7: 211-224.

19. Nelson CM, Bissell MJ (2006) Of extracellular matrix, scaffolds, and signaling tissue architecture regulates development, homeostasis, and cancer. Annu Rev Cell Dev Biol 22: 287-309.

20. Minchinton Al, Tannock IF (2006) Drug penetration in solid tumours. Nat Rev Cancer 6: 583-92. 